\title{
Brittle Porous Material Mesovolume Structure Models and Simulation of their Mechanical Properties
}

\author{
I. Yu. Smolin 1, 2, a) , M. O. Eremin 1,2, b), P. V. Makarov ${ }^{1,2, c),}$

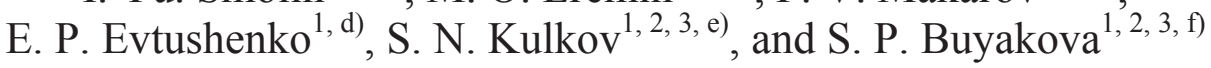 \\ ${ }^{1}$ Institute of Strength Physics and Materials Science SB RAS, Tomsk, 634055, Russia \\ ${ }^{2}$ National Research Tomsk State University, Tomsk, 634050, Russia \\ ${ }^{3}$ National Research Tomsk Polytechnic University, Tomsk, 634050, Russia

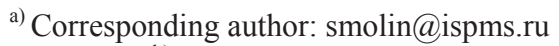 \\ b) bacardi@ispms.ru \\ ${ }^{c)}$ pvm@ispms.ru \\ d) eugene@ispms.ru \\ e) kulkov@ispms.ru \\ f) sbuyakova@ispms.ru
}

\begin{abstract}
To study the mechanical response of brittle porous materials at mesoscale, porous samples were generated and their deformation was numerically modelled. Two types of pore space morphology such as overlapping spherical pores and overlapping spherical solids were explicitly considered. For deformation modelling, an evolutionary approach including the nonlinear constitutive equations used to describe damage accumulation and its impact on the degradation of the solid frame strength properties was applied. The numerical results have shown that an average stress-strain diagram is sensitive to pore morphology as well as porosity.
\end{abstract}

Keywords: ceramics, porous structure, modelling, structure - property relationship

\section{INTRODUCTION}

The problems of porous materials modelling are often discussed in literature, e.g. [1-4]. They can be divided into three areas: (i) modelling the porous structure per se; (ii) determining the effective properties of porous materials; (iii) direct numerical simulation of deformation and fracture of porous materials at different scales. The purpose of this paper is to discuss approaches to modelling (i) porous structure of real materials (a geometric problem) and (ii) mechanical behaviour (deformation, damage accumulation and fracture) of porous materials mesovolumes.

As usual, porous materials are hierarchically organized multiscale systems. A basic simulation problem of such materials is the construction of the constitutive equations describing their deformation response, and especially the failure. The evolutionary methodology, developed by the authors [5-7], is an effective approach to solving the problem. A material under loading is considered as a nonlinear dynamic multiscale system in which the nonlinear positive and negative feedbacks are explicitly specified regulating the interaction between the stress-strain state emerging in the material and its response to loading. Such an approach can be used to effectively describe the evolution of the porous materials properties at different scales including the localized damage accumulation and inelastic deformation at micro- and mesoscales, strength degradation, different scale cracks formation and the macroscopic failure (main crack formation).

\section{GEOMETRIC MODELS OF THE POROUS MEDIUM}

Following [1, 2], two different morphologies of the porous structure statistical models: overlapping spherical pores and overlapping solid spheres are considered. In the first case, a porous medium model represents a sample of International Conference on Physical Mesomechanics of Multilevel Systems 2014
AIP Conf. Proc. 1623, 595-598 (2014); doi: 10.1063/1.4899015

(C) 2014 AIP Publishing LLC 978-0-7354-1260-6/\$30.00 
a solid comprising spherical voids of different radii at random (uncorrelated) points in space. This model mimics the morphology of isolated pores in realistic materials at low porosity caused by the processes of coalescence and spheroidization of pores, e.g. in ceramics at the final stage of sintering. In the second case, the geometric model is constructed by filling an empty space by solid spherical particles of different radii, which are placed at the randomly selected points in space and may overlap. This model correlates with the process of sintering of ideal spherical particles, e.g. ceramic powder.

We generated a few geometric structures consisting of voxels representing two types of pore morphology considered above. Two of them are shown in Fig. 1(a,b). These voxel structures were used as an input for the following computer simulation of their deformation and fracture. To estimate how these model structures mimic reality, one can compare them with the photo of the porous zirconia ceramics surface illustrated in Fig. 1(c). The ceramics was obtained by sintering of nanostructured zirconia powders [8]. It is seen that in realistic ceramics there are pores of different morphologies in one and the same material. This is due to various factors such as changing the process conditions, different forms of individual powder particles, etc.

Substantial interest has arisen in the numerical investigation of the ideal porous structures.

\section{THE FRAMEWORK OF THE EVOLUTIONARY APPROACH TO MODELLING DEFORMATION AND FAILURE}

In accordance with the concept of the evolutionary description of deformation and subsequent fracture of materials $[5,6]$, a complete set of equations in the Lagrangian description of the continuum motion includes the fundamental laws of mass, momentum and energy conservation, geometric relationships between strain rate and velocity, and two groups of the constitutive equations. The evolutionary constitutive equations of the first group define the relationship between stress and strain in the relaxation (rate) form. Since total strain is the sum of elastic and inelastic parts, the rate form of the constitutive equations describes the stress increase caused by the increase in total strains and stress relaxation due to the development of inelastic strain. The concern of the evolutionary equations of the second group is to determine the rate of inelastic strains in the first group of the constitutive equations. Here, strain rate is determined by the plastic flow theory. Limiting stress surface is written in the von Mises-Schleicher form with taking into account the dependence of the shear strength on the pressure, which in turn results in different tensile and compressive strength of the material. A non-associated flow rule is used, which allows to describe dilatation as independent of internal friction. In this case, the plastic potential is different from the yield function.

The failure of a material in this approach is described as the process of downfall degradation of the material strength to zero when crack formation occurs at the ultrafast catastrophic stage of the stress-strain state evolution. This stage follows the pre-fracture stage where gradual damage accumulation takes place. The features of the damage accumulation play a crucial role in the transition of the medium strength evolution from the steady deformation to the catastrophic super-fast mode.
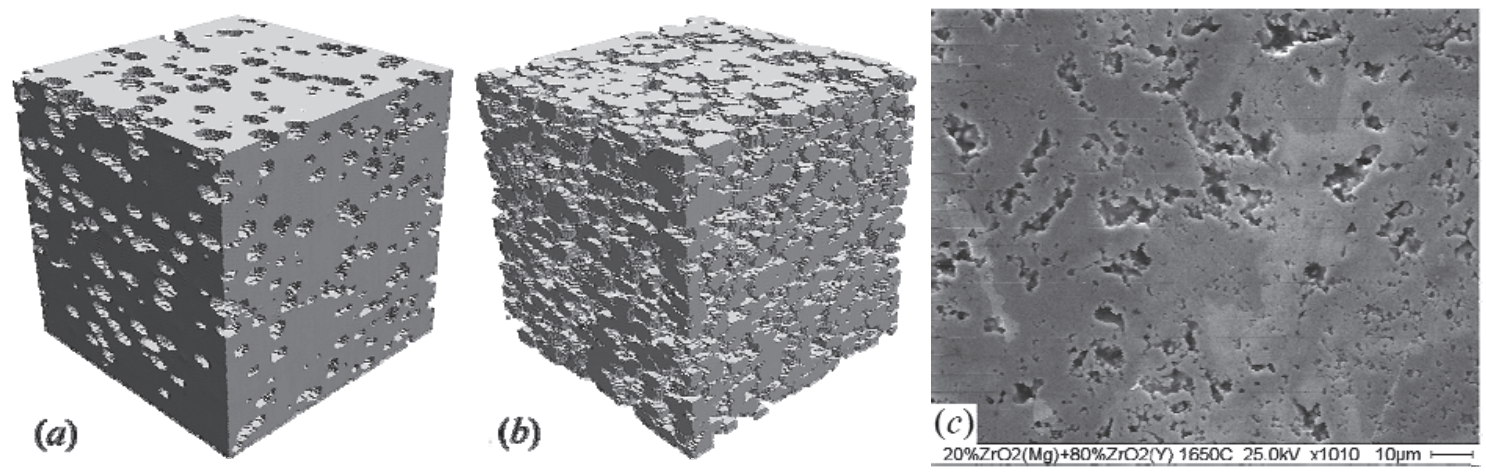

FIGURE 1. 3D model structures having different pore morphology: (a) overlapping spherical pores structure,

(b) overlapping solid spheres structure, and (c) the photo of the porous structure in zirconia ceramics 
The medium degradation (damage) is represented here as a function of accumulated inelastic strain and stress state type (Lode-Nadai parameter $\mu_{\sigma}$ ) so that damage accumulation begins at substantially lower stress in the areas of tension-shear $\left(\mu_{\sigma}<0\right)$ than in the areas of compression-shear where $\mu_{\sigma}>0$. The damage accumulation rate in the local areas where $\mu_{\sigma}<0$ is also significantly higher. Thus, the medium response to the type of stress state (its current strength) is yielded in the medium during its loading. As a result, the strength parameters will degrade much faster in those regions of the medium where the Lode-Nadai parameter is negative. The detailed description of the mathematical model is given in [7].

\section{CALCULATION RESULTS AND DISCUSSION}

The simulation of inelastic deformation and fracture of porous quasi-brittle media were performed by solving the total set of equations in a three-dimensional formulation using the finite difference scheme described in detail in [9].

Modelling the mechanical behaviour of the porous materials generated model structures was carried out for the conditions of uniaxial compression. Calculations were performed using the isotropic material properties which correspond to the partially stabilized zirconia in the tetragonal phase. Values for the parameters of damage accumulation kinetics were taken from the test calculation to produce the features of fracture observed in the experiments. The size of the computational domain was always $9 \times 9 \times 9 \mu \mathrm{m}^{3}$. The uniform computational grid consisted of $150 \times 150 \times 150$ nodes. Cells representing the pores were modelled by an effective elastic material with mass and elastic properties of several orders of magnitude smaller than the corresponding characteristics of ceramics.

Figure 2(a) shows the average stress-strain curves for four different samples. One can see that the lower is porosity the higher is corresponding curve that agrees with the experimental observations. For the same porosity, the curve for overlapping solid spheres morphology lies below. Similar information has been reported in $[1,2]$ for the purely elastic behaviour. Besides, according to [2], the dependence of Young's modulus $E$ on the average porosity $p$ for different morphologies has the following form: $E=E_{\mathrm{d}}(1-p)^{m}$, where $E_{\mathrm{d}}$ is the Young's modulus of a dense material (or solid domain), and the index of power $m=2$ for the overlapping spherical pores structures and $m=4$ for overlapping solid spheres structures. The experimental studies of porous zirconia with the porosity varying in the range from $10 \%$ to $75 \%$ and different ratio of pore size and ceramics grain size indicate that the dependence of the Young's modulus on the porosity is better approximated by the exponential function $E=E_{\mathrm{d}} \exp (-b p)$. Comparison of the analytical power and the exponential experimental dependencies, as well as our own calculations data are shown in Fig. 2(b). It is seen that the exponential dependence and the power dependence are very close for overlapping solid spheres morphology in the selected range of porosity. The difference between them for small, and especially for large porosity can be explained by the fact that pore morphology of the experimental samples does not correspond strictly to the overlapping spherical solids. The results data obtained in our calculations are also in a good agreement with the power law for overlapping solid spheres morphology.
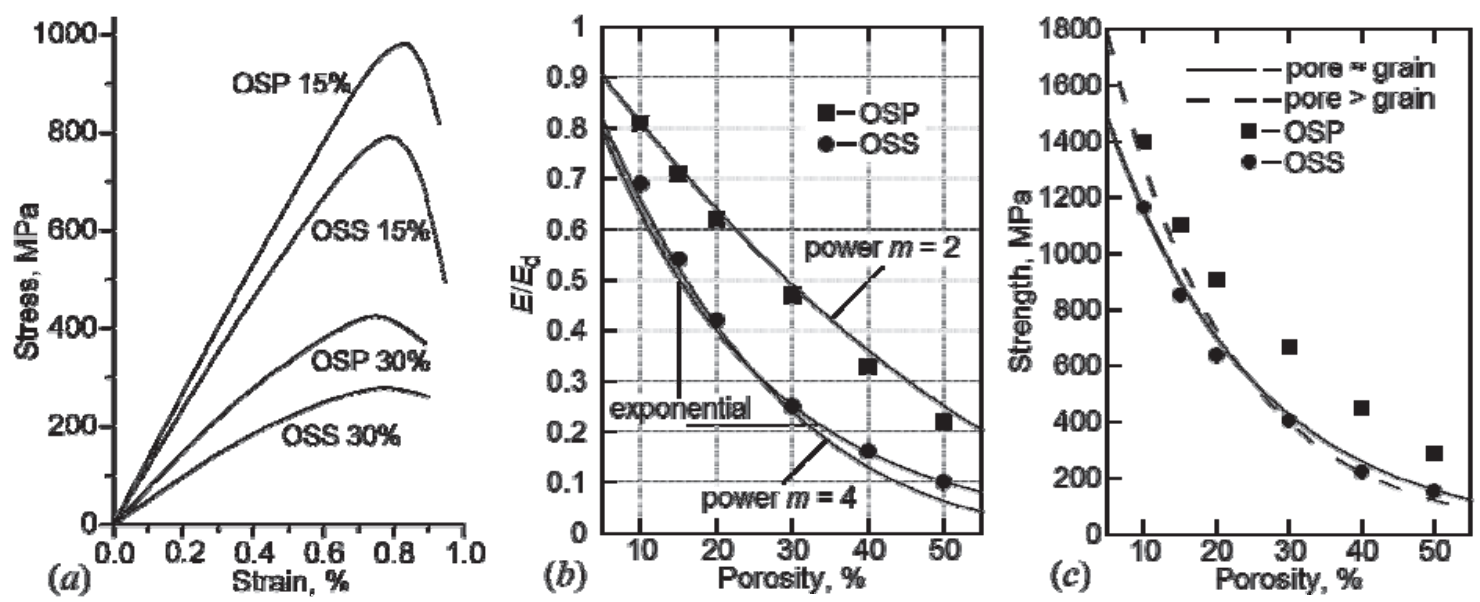

FIGURE 2. Computational results: (a) stress-strain curves for different morphologies and porosity; (b) the dependence of reduced effective modulus of elasticity for porous samples in comparison with analytical and experimental data; (c) the influence of porosity on the compressive strength of porous samples in comparison with the experimental data 
The dependence of strength characteristics on the porosity is also of special interest. The experimental data for zirconia ceramics were also approximated using an exponential function with different parameters depending on the commensurability of sizes of pores and grains. Figure 2(c) shows these approximating curves and points obtained in our calculations. The calculated data for overlapping solid spheres morphology again are in very good agreement with the experimental curves.

\section{CONCLUSION}

Our calculations have shown that the model structures created by combining overlapping spherical solids well mimic the porous ceramic materials produced by sintering of oxide powders. These geometric models better meet

pore morphology of porous zirconia ceramics real samples and are in good agreement with the dependence of the average Young's modulus and strength on the total porosity when used in modelling the mechanical behaviour. Some disagreement of the calculation results with the experimental data should be explained by the fact that in the model of porous structures the pores are distributed quasiuniformly, while in the reality the pore clustering is observed. The simulation with varying degrees of clustering is the goal of a future research.

The application of the evolutionary approach, in which the regions of localized damage are generated at the stage of macroscopic pre-fracture, has provided a correct description of the formation of high damage (cracks) areas at the mesoscale, and the degradation influence in local regions of the material on the general nature of the stress-strain diagram including its descending branch. The further macroscopic failure develops as a catastrophe in the blow-up regime.

\section{ACKNOWLEDGEMENT}

This research was supported by the Program of basic scientific research of Russian Academy of Sciences in 2013-2020. Calculations were performed on SKIF Cyberia supercomputer at Tomsk State University.

\section{REFERENCES}

1. A. Roberts and E. Garboczi, J. Am. Ceram. Soc. 83, 3041 (2000).

2. G. Bruno, A. M. Efremov, A. N. Levandovskyi, and B. Clausen, J. Mater. Sci. 46, 161 (2011).

3. Ig. S. Konovalenko, A. Yu. Smolin, and S. G. Psakhie, Frattura ed Integrità Strutturale 24, 75 (2013).

4. A. Yu. Smolin, N. V. Roman, I. S. Konovalenko, G. M. Anikeeva, and S. G. Psakhie, Engng Fract. Mech. (2014), http://dx.doi.org/10.1016/j.engfracmech.2014.04.001 (in press).

5. P. V. Makarov, Phys. Mesomech. 11(5-6), 213 (2008).

6. Yu. A. Kostandov, P. V. Makarov, M. O. Eremin, I. Yu. Smolin, and I. E. Shipovskii, Int. Appl. Mech., 1, 95 (2013).

7. P. V. Makarov and M. O. Eremin, Phys. Mesomech. 16(3), 207 (2013).

8. S. P. Buyakova and S. N. Kulkov, Nanotech. Russ. 1, 119 (2007).

9. M. L. Wilkins, Computer Simulation of Dynamic Phenomena (Springer-Verlag, Berlin, 1999). 